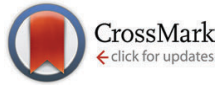

Cite this: Phys. Chem. Chem. Phys., 2015, 17, 9116

Received 6th November 2014, Accepted 10th February 2015

DOI: $10.1039 / c 4 c p 05148 c$

www.rsc.org/pccp

\section{Tailoring the optical properties of lanthanide phosphors: prediction and characterization of the luminescence of $\mathrm{Pr}^{3+}$-doped $\mathrm{LiYF}_{4} \dagger$}

\author{
Harry Ramanantoanina, ${ }^{* a}$ Werner Urland, ${ }^{a}$ Benjamin Herden, ${ }^{a}$ Fanica Cimpoesu ${ }^{b}$ \\ and Claude Daula
}

\begin{abstract}
We present a theoretical work detailing the electronic structure and the optical properties of $\left(\operatorname{PrF}_{8}\right)^{5-}$ embedded in $\mathrm{LiYF}_{4}$, complementing the insight with data that are not available by experimental line. The local distortions due to the embedding of the lanthanide ion in the sites occupied in the periodic lattice by smaller yttrium centres, not detectable in regular X-ray analyses, are reproduced with the help of geometry optimization. Then, based on the local coordination environment, the relation structure-optical properties is constructed by Density Functional Theory computations in conjunction with the ligand field theory analyses $\left(\right.$ LFDFT) determining the $[\mathrm{Xe}] 4 \mathrm{f}^{2} \rightarrow[\mathrm{Xe}] 4 \mathrm{f}^{1} 5 \mathrm{~d}^{1}$ transitions. In previous instances we analysed rather symmetric systems, here facing the complexity of low symmetry cases, treated in the Wybourne ligand field parameterization and in the Angular Overlap Model (AOM) frame. A very important improvement at the AOM level is the consideration of the $f-d$ mixing that brings coupling term of odd-even nature, essential for the realistic description of the asymmetric coordination centres. Furthermore, we introduce now a principle for modelling the emission intensity. The results are in agreement with available experimental findings. The relevance of the modelling has a practical face in the rational design of optimal luminescent materials needed in domestic lightening and also an academic side, revisiting with modern computational tools areas incompletely explored by the standard ligand field theories.
\end{abstract}

\section{Introduction}

The recent award of a Nobel prize for the invention of blue light-emitting diodes ${ }^{1}$ has enhanced the interest in the role already played and further engineering related to this class of devices. An important application concerns the less consuming lighting. In this case the blue main component should be complemented with wavelengths obtained from coating materials, tuning the light toward the solar day spectrum, the so-called warm-white light. Typical coating materials involve lanthanide ions, like the popular $\mathrm{Y}_{3} \mathrm{Al}_{5} \mathrm{O}_{12}$ doped with $\mathrm{Ce}^{3+1}$, taking advantage of the optical properties of the $\mathrm{f}-\mathrm{d}$ transitions. Briefly described, the active centers for the luminescent coating (the phosphors) absorb high energy photons from the violet or the ultraviolet range of the basic source, the light-emitting diode (LED),

\footnotetext{
${ }^{a}$ Department of Chemistry of the University of Fribourg, Chemin du Musée 9, 1700 Fribourg, Switzerland. E-mail: harry.ra@hotmail.com, claude.daul@unifr.ch; Fax: +41 26300 9738; Tel: +41 263008700

${ }^{b}$ Institute of Physical Chemistry, Splaiul Independentei 202, Bucharest 060021, Romania.E-mail: cfanica@yahoo.com

$\dagger$ Electronic supplementary information (ESI) available: Numerical data for the multiplet energy levels given in Fig. 4 and 5 obtained for the $[\mathrm{Xe}] 4 \mathrm{f}^{2}$ and $[\mathrm{Xe}] 4 \mathrm{f}^{1} 5 \mathrm{~d}^{1}$ electron configurations of $\mathrm{Pr}^{3+}$ in LiYF 4 : $\mathrm{Pr}^{3+}$. See DOI: 10.1039/c4cp05148c
}

undergoing after that a stepwise de-excitation and releasing radiation with smaller energy that contributes to the desired alleviation of the overall emitted spectrum. Several lanthanide ions are good candidates for phosphors, which apart from improving the emission profile, play the role of improving the energy yield, transmuting light from the invisible range to the domain appropriate for human eye sensitivity.

The $\mathrm{Pr}^{3+}$ ion is a potential activator for modern LED phosphors. It provides the red emission important for the quest for warm-white LED lighting, ${ }^{2}$ and its electronic structure enables intriguing optical manifestation, for instance the photoncascade emission. ${ }^{3-5}$ The $\mathrm{Pr}^{3+}$-doped $\mathrm{LiYF}_{4}$ is also a well studied system applied for laser materials. ${ }^{6,7}$ For the design of modern LED phosphors, it is of crucial importance to predict the electronic structure and the relation with optical properties to help the synthesis in laboratories by useful thumb rules. The theoretical modeling brings a better understanding of the microscopic origin of the optical phenomenon. In $\operatorname{Pr}^{3+}$ phosphors, the problem is settled with respect to the ground $[\mathrm{Xe}] 4 \mathrm{f}^{2}$ and the excited $[\mathrm{Xe}] 4 \mathrm{f}^{1} 5 \mathrm{~d}^{1}$ electron configurations. ${ }^{8}$ Several quantum chemical methods may in principle be used to tackle the problem. ${ }^{9-11}$ The Ligand Field Density Functional Theory (LFDFT) approach ${ }^{8}$ is convenient because of its relative simplicity and the chemical 
intuitiveness of its results. A plus of transparency is gained using as ligand field part the angular overlap model (AOM), ${ }^{12,13}$ whose parameters are understandable as well-categorized ligand contributions. Special attention should be devoted to the different rates of the nephelauxetic effect in $\mathrm{f}$ versus $\mathrm{d}$ shells, ${ }^{14}$ such details being well tracked along the LFDFT algorithm. One must also point that the LFDFT relies on specific features offered by the Amsterdam Density Functional (ADF) code ${ }^{15-17}$ enabling the control of orbital population and generating non-aufbau occupation schemes.

Ligand field theory is based on a phenomenological Hamiltonian (eqn (1)) considering the perturbation of the metal center limited to the basis of the partially filled $\mathrm{f}$ or $\mathrm{d}$ atomic orbitals. ${ }^{18,19}$ A two-open-shell ligand field theory, as in our case considering both the $\mathrm{f}$ and d shells, is a rather special variety, necessary to develop treatments of the enounced type, dealing with inter-shell spectroscopy. The whole Hamiltonian is built considering three important interactions, such as the inter-electron repulsion $\left(H_{\mathrm{EE}}\right)$, the ligand field potential $\left(V_{\mathrm{LF}}\right)$ and the spin-orbit coupling interaction $\left(H_{\mathrm{SO}}\right)$. The respective interactions are parameterized in terms of the Slater-Condon integrals, the ligand field one-electron part and the spin-orbit coupling constants. Since the inter-electron and spin-orbit part are mostly confined to the free atom modeling, the key role is carried by the ligand field part. The ligand field theory is nowadays useful in multifarious aspects of inorganic chemical science, both in theoretical investigations $s^{8,9,11,20-27}$ as well as experimental work. ${ }^{28,29}$

$$
H=H_{0}+H_{\mathrm{EE}}+V_{\mathrm{LF}}+H_{\mathrm{SO}}
$$

In eqn (1), $H_{0}$ is a diagonal matrix, which includes the energy shift between states from the $[\mathrm{Xe}] 4 \mathrm{f}^{1} 5 \mathrm{~d}^{1}$ and the $[\mathrm{Xe}] 4 \mathrm{f}^{2}$ configurations. This energy gap is parameterized by the $\Delta(\mathrm{fd})$ parameter, discussed in precedent work. ${ }^{8}$

Herein we present a theoretical prediction of the electronic structure and optical properties of $\mathrm{LiYF}_{4}: \operatorname{Pr}^{3+}$ via LFDFT calculations of the multiplet energy levels arising from the ground $[\mathrm{Xe}] 4 \mathrm{f}^{2}$ and the excited $[\mathrm{Xe}] 4 \mathrm{f}^{1} 5 \mathrm{~d}^{1}$ electron configurations of $\mathrm{Pr}^{3+}$ in a certain chemical environment. We validate the theoretical model taking into account aspects from several experimentally available investigations. Furthermore we include an intensity determination based on zero-phonon lines, advancing toward a more realistic description and simulation of the optical features.

\section{Methodology}

In this work, the DFT calculations have been carried out by means of the ADF program package (ADF2013.01). ${ }^{15-17}$ We use the hybrid B3LYP functional, as it is implemented in the ADF program package ${ }^{15-17}$ for the exchange and correlation energy and potential, to compute the electronic structure and the related optical properties, in line with previous work. ${ }^{8}$ However we use the pure local density approximation (LDA) functional and the generalized gradient approximation (GGA) functional for the geometry optimization. The molecular orbitals are expanded using triple-zeta plus two polarization Slater-type orbital (STO) functions (TZ2P+) for the Pr atom and triplezeta plus one polarization STO function (TZP) for the F, Y and Li atoms. The LFDFT designates computation experiments and post-calculation analyses meant to exploit the DFT calculations in the sense of ligand field theory. This is possible in codes allowing the handling of orbital and spin occupations, generating non-aufbau occupation schemes. The artificial configurations can be regarded in a manner similar to Broken-Symmetry (BS) DFT procedures in relation to the estimation of exchange coupling effects: the BS-DFT configurations are not real states but are objects from where parametric information can be extracted. ${ }^{30-32}$ The LFDFT implies generating different configurations inside a DFT calculation engine, treated non-iteratively on the basis of orbitals obtained in the conditions of the so-called Average-of-Configuration (AOC) type calculation, followed by the fit of the numeric results in accordance with the Ligand Field master formula in the given frame of the assumed parametric scheme. ${ }^{18,19,27}$ The states are not excited states, but artificial determinants containing the needed information. The AOC consists in imposing fractional occupation numbers on molecular orbitals assigned to d or f parentage, by smearing to $n / 5$ and $m / 7$, respectively, the electron count of $\mathrm{d}^{n}, \mathrm{f}^{m}$ or $\mathrm{d}^{n} \mathrm{f}^{m}$ configurations. Considering that fractional occupations are allowed in DFT, ${ }^{33}$ the AOC produces optimized orbitals closer to the ligand field meaning, where a spherically averaged reference is assumed especially in the account of two-electron terms by the use of Slater-Condon parameters in a formalism resembling the free ion case.

The non-doped crystal structure from the X-ray diffraction data of the $\mathrm{LiYF}_{4}$ is obtained from ref. 34. Then, a $\mathrm{Pr}^{3+}$ ion is introduced, replacing one $\mathrm{Y}^{3+}$ ion. The geometry of the doped system is hardly obtained from the experimental studies. In turn it can be simulated by means of the periodical crystal structure approach based on a super-cell model. ${ }^{35,36}$ A geometry optimization based on the molecular cluster approach can also be considered requiring a specific restricted region of the crystal structure. This molecular cluster approach of the local crystal environment may look modest with respect to geometry optimization goals. However in previous instances ${ }^{35}$ we found that it reproduces well the full super-cell band structure calculations. ${ }^{35}$ The molecular cluster procedure is demanded by a special branch of treatment, concerning the geometries of excited state configurations, which so far cannot be addressed by common band structure algorithms. This gives further insight into the investigation of vibronic coupling effects $^{37,38}$ or Stokes shift. ${ }^{39}$

From the crystal structure of $\mathrm{LiYF}_{4},{ }^{34}$ we pick one $\mathrm{Y}^{3+}$ center together with any chemical elements located inside the sphere of constant radius of $4.5 \AA$ around this center. This yields a cluster of thirty-seven atoms, i.e. five $\mathrm{Y}^{3+}$, eight $\mathrm{Li}^{+}$and twentyfour $\mathrm{F}^{-}$, where the global charge is exactly -1 . The small negative charge is a compromise for keeping the cluster at an initial symmetry corresponding to the yttrium-site, since adding a further lithium ion, for instance to bring neutrality, will introduce a bias into the optimization procedure. In the cluster model, the central $\mathrm{Y}^{3+}$, which will be doped with $\mathrm{Pr}^{3+}$, is eightfold coordinated by fluoride ligands whereas for the remaining 


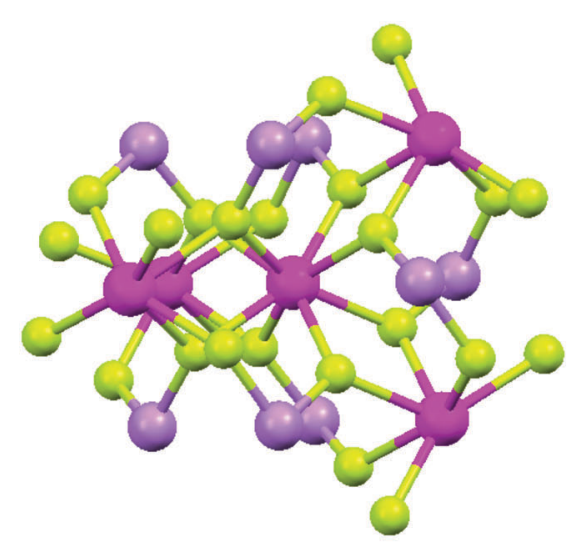

(a)

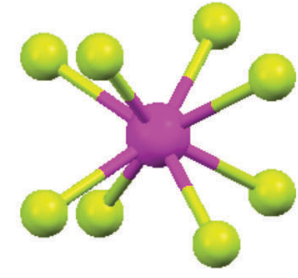

(b)

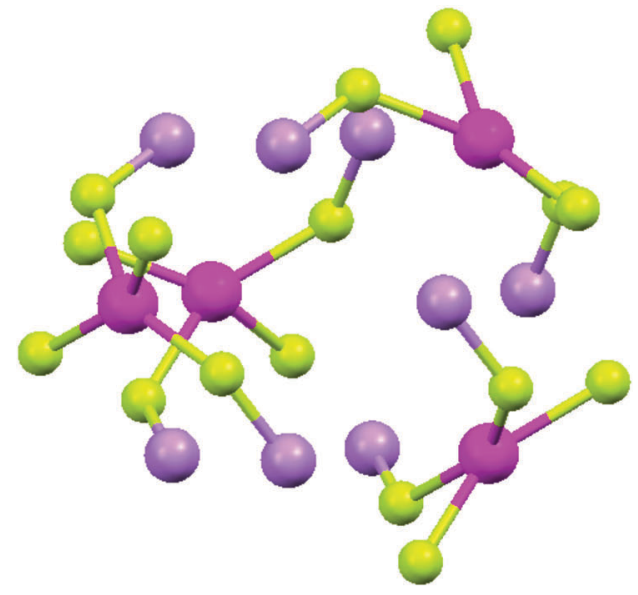

(c)

Fig. 1 Spatial representations of the selected cluster from the LiYF 4 bulk used in the calculation. There are thirty-seven atoms forming the whole cluster (a) which can be observed as $\left(\mathrm{YF}_{8}\right)^{5-}$ (b) embedded in a chemical environment (c). Colour code: $\mathrm{Y}^{3+}$ (magenta), $\mathrm{Li}^{+}$(violet) and $\mathrm{F}^{-}$(green).

four $\mathrm{Y}^{3+}$ ions only six ligands are included because of charge compensation. The eight $\mathrm{Li}^{+}$ions are on the other hand coordinated by two fluoride ligands. A graphical representation of this cluster is given in Fig. 1. The geometry optimization proceeds separating the cluster (Fig. 1a) in two distinct regions: one moiety (Fig. 1b) where the geometry optimization is performed and another one (Fig. 1c) whose coordinates are kept frozen to the experimental crystal structure. In this way we focus our interest only in the local changes of the structure of $\left(\mathrm{PrF}_{8}\right)^{5-}$ embedded in $\mathrm{LiYF}_{4}$. We use the structure of $\left(\mathrm{PrF}_{8}\right)^{5-}$ obtained in this geometry optimization as input for the determination of the electronic structure and properties we are looking for. We use the methodology illustrated in ref. 8 taking as basis 231 Slater-Determinants energies from the ground $[\mathrm{Xe}] 4 \mathrm{f}^{2}$ (91 microstates) and the excited $[\mathrm{Xe}] 4 \mathrm{f}^{1} 5 \mathrm{~d}^{1}$ (140 microstates) configurations of $\mathrm{Pr}^{3+}$. The ligand field potential $\left(V_{\mathrm{LF}}\right)$ is fitted from these Slater-Determinants energies and the $\Delta(\mathrm{fd})$ parameter is the difference of the barycenters of the energies of the Slater-Determinants of the excited $[\mathrm{Xe}] 4 \mathrm{f}^{1} 5 \mathrm{~d}^{1}$ and the ground $[\mathrm{Xe}] 4 \mathrm{f}^{2}$ configurations. This $\Delta(\mathrm{fd})$ parameter is algebraically evaluated in the following:

$$
\Delta(\mathrm{fd})=h_{\mathrm{d}}-h_{\mathrm{f}}+F_{0}(\mathrm{fd})-F_{0}(\mathrm{ff})+B_{0}^{0}(\mathrm{~d})-B_{0}^{0}(\mathrm{f}),
$$

involving contributions from terms appropriate to the free ion: $h_{\mathrm{d}}, h_{\mathrm{f}}$ and the zeroth-order Slater-Condon $F_{0}(\mathrm{ff})$ and $F_{0}(\mathrm{fd})$ parameters as well as the diagonal elements of the ligand field potential $B_{0}^{0}(\mathrm{f})$ and $B_{0}^{0}(\mathrm{~d})$, which cannot and do not need to be discriminated separately.

The Slater-Condon $F_{k}(\mathrm{ff}), F_{k}(\mathrm{fd})$ and $G_{k}(\mathrm{fd})(k \neq 0)$ parameters are obtained from the radial functions of the $4 \mathrm{f}$ and $5 \mathrm{~d}$ Kohn-Sham orbitals of $\mathrm{Pr}^{3+}$ according to eqn (3)-(5), respectively, whose procedure is already described in ref. 36.

$$
\begin{aligned}
& F_{k}(\mathrm{ff})=\int_{0}^{\infty} \int_{0}^{\infty} \frac{r_{<}^{k}}{r_{>}^{k+1}} R_{4 \mathrm{f}}^{2}\left(r_{1}\right) R_{4 \mathrm{f}^{2}}{ }^{2}\left(r_{2}\right) r_{1}^{2} r_{2}^{2} \mathrm{~d} r_{1} \mathrm{~d} r_{2}, \\
& F_{k}(\mathrm{fd})=\int_{0}^{\infty} \int_{0}^{\infty} \frac{r_{<}^{k}}{r_{>}^{k+1}} R_{4 \mathrm{f}}^{2}\left(r_{1}\right) R_{5 \mathrm{~d}}{ }^{2}\left(r_{2}\right) r_{1}^{2} r_{2}^{2} \mathrm{~d} r_{1} \mathrm{~d} r_{2},
\end{aligned}
$$

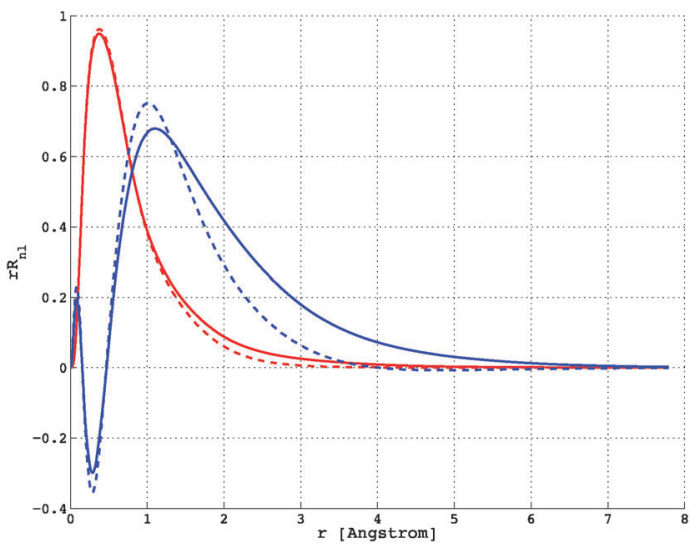

Fig. 2 Graphical representation of the radial functions of the $4 f$ (in red) and $5 \mathrm{~d}$ (in blue) Kohn-Sham orbitals of $\mathrm{Pr}^{3+}$ for the inter-configuration $4 \mathrm{f} / 5 \mathrm{~d}$ interactions in the free ion (dashed line) and in $\left(\mathrm{PrF}_{8}\right)^{5-}$ embedded in $\mathrm{LiYF}_{4}$ (solid line).

$$
G_{k}(\mathrm{fd})=\int_{0}^{\infty} \int_{0}^{\infty} \frac{r_{<}^{k}}{r_{>}^{k+1}} R_{4 \mathrm{f}}\left(r_{1}\right) R_{5 \mathrm{~d}}\left(r_{2}\right) R_{5 \mathrm{~d}}\left(r_{1}\right) R_{4 \mathrm{f}}\left(r_{2}\right) r_{1}^{2} r_{2}^{2} \mathrm{~d} r_{1} \mathrm{~d} r_{2}
$$

where $R_{4 \mathrm{f}}$ and $R_{5 \mathrm{~d}}$ are the radial functions of the $4 \mathrm{f}$ and $5 \mathrm{~d}$ Kohn-Sham orbitals of $\operatorname{Pr}^{3+}$ (cf. Fig. 2); $r_{<}$and $r_{>}$are the lesser and bigger of $r_{1}$ and $r_{2}$, respectively.

The spin-orbit coupling constants $\zeta_{4 \mathrm{f}}$ and $\zeta_{5 \mathrm{~d}}$ are calculated using the approach of ZORA relativistic available in the ADF program package. ${ }^{15-17}$

\section{Results and discussion}

Before entering into the ligand field problem, the simulated geometry of the $\left(\mathrm{YF}_{8}\right)^{5-}$ fragment embedded in $\mathrm{LiYF}_{4}$ is discussed. The $\mathrm{LiYF}_{4}$ crystallizes in the I41/a (No. 88) space group, where the Wyckoff position of the yttrium-site has a $S_{4}$ environment. In this $S_{4}$ coordination, the eight fluoride ligands surrounding 
Table 1 Experimental and calculated structures of $\left(\mathrm{YF}_{8}\right)^{5-}$ and $\left(\mathrm{PrF}_{8}\right)^{5-}$ embedded in LiYF 4 : the polar coordinates $d_{1}$ (in $\AA$ ), $\theta_{1}$ and $\phi_{1}$ (in ${ }^{\circ}$ ); $d_{2}$ (in $\AA$ ), $\theta_{2}$ and $\phi_{2}\left(\right.$ in $^{\circ}$ ) of two fluoride ligands from which the rest of the structure is generated by symmetry

\begin{tabular}{llllllll}
\hline & $\left(\mathrm{YF}_{8}\right)^{5-}$ & & & & & & \\
\cline { 2 - 4 } & Exp. $^{a}$ & $\mathrm{X} \alpha$ & $\mathrm{PWN}$ & ${ }^{5-}$ \\
\hline$d_{1}$ & 2.246 & 2.2479 & 2.2468 & 2.2650 & 2.2565 & 2.3425 \\
$\theta_{1}$ & 67.05 & 66.58 & 66.57 & 66.31 & 66.44 & 65.62 \\
$\phi_{1}$ & -33.00 & -35.79 & -35.68 & -36.81 & -36.76 & -37.86 \\
$d_{2}$ & 2.293 & 2.3031 & 2.3012 & 2.3217 & 2.3234 & 2.3967 \\
$\theta_{2}$ & 142.03 & 139.34 & 139.39 & 138.54 & 138.63 & 137.39 \\
$\phi_{2}$ & -36.59 & -36.51 & -36.59 & -36.06 & -35.92 & -36.18 \\
dev. $^{b}$ & - & 0.1900 & 0.1831 & 0.2650 & 0.2628 & -
\end{tabular}

${ }^{a}$ These values are taken from ref. 40 and $41 .{ }^{b} \mathrm{dev}$. (in $\AA$ ) stands for the calculated mean deviations between the optimized structure (Cartesian coordinates) of $\left(\mathrm{YF}_{8}\right)^{5-}$ and the experimental data.

the $\mathrm{Y}^{3+}$ are four by four identical (Fig. 1b). Their symmetry distinct ligand coordinates can be generated by six polar coordinates, $d_{1}, \theta_{1}, \phi_{1}, d_{2}, \theta_{2}$ and $\phi_{2}$, whose experimental values are given in Table 1, collected from ref. 40 and 41. A geometry optimization of $\left(\mathrm{YF}_{8}\right)^{5-}$ embedded in $\mathrm{LiYF}_{4}$ is performed by means of DFT calculations, based on different exchange and correlation functionals, i.e. the LDA: $\mathrm{X} \alpha^{42}$ and Vosko-WilkNussair (VWN $)^{43}$ as well as the GGA: Becke-Perdew (BP86) ${ }^{44,45}$ and PW91. ${ }^{46}$ We proceed in this way to make explicit which DFT functional is better appropriate for the structural determination. Table 1 reports the values of the experimental and calculated structures using polar coordinates representation. In general, the deviations with the experimental data are rather small considering the four DFT functionals (Table 1). We can discriminate the influence of the DFT setting in reporting a slight elongation of the $\mathrm{Y}-\mathrm{F}$ bond lengths using the GGA formalism, i.e. BP86 and PW91 (Table 1). However the calculations based on the LDA formalism, i.e. $\mathrm{X} \alpha$ and VWN, offer the best match to the experimental results (Table 1), a fact that has been already recognized before. ${ }^{35,47}$

We consider explicitly here the VWN functional for any structural determination. Therefore the structure of $\left(\mathrm{PrF}_{8}\right)^{5-}$ embedded in $\mathrm{LiYF}_{4}$ is determined and the coordinates are presented in Table 1. It is worth mentioning that while the $\left(\mathrm{YF}_{8}\right)^{5-}$ is a closed-shell cluster, the $\left(\mathrm{PrF}_{8}\right)^{5-}$ has an open-shell electronic structure due to the presence of two valence electrons in the $4 \mathrm{f}$ orbitals of $\mathrm{Pr}^{3+}$, leading to a non-trivial issue in the optimization of the geometry. The rational way is to impose the average of configuration (AOC) type calculation, ${ }^{48-50}$ i.e. equal smearing of the two valence electrons into the seven-fold $4 \mathrm{f}$ orbitals of $\mathrm{Pr}^{3+}$, amending the tedious issue due to possible divergence in the self-consistent field (SCF) electronic structure. This is in line with the conceptual frame of ligand field theory, relying on an averaged reference which is fully equivalent, in technical respects, to the AOC procedure. Moreover the $4 \mathrm{f}$ orbitals being shielded by the outer shells in the lanthanide system, the $4 \mathrm{f}$ electrons are not participating in the chemical bonding. Thus a specific population of the two electrons in the 4f orbitals will not affect the geometry of $\left(\mathrm{PrF}_{8}\right)^{5-}$ like it is explicitly stated in ref. 35 for the case of $\mathrm{Ce}^{3+}$ compounds.
We specifically notice an elongation of the Pr-F bond lengths, although the polar and azimuthal angles remain similar to the $\left(\mathrm{YF}_{8}\right)^{5-}$ (Table 1), in agreement with the Shannon radii ${ }^{51}$ of $\operatorname{Pr}^{3+}$ and fluoride ligand in such an eight coordination.

The LFDFT treatment is performed on the cluster $\left(\mathrm{PrF}_{8}\right)^{5-}$ according to the structure given in Table 1, where the positions of the next nearest neighbouring $\mathrm{Li}^{+}$and $\mathrm{Y}^{3+}$ ions (Fig. 1) are used as point charges to neutralize the high negative charge of the cluster. The ligand field treatment works on the basis of $\mathrm{LM}_{\mathrm{L}} \mathrm{SM}_{\mathrm{S}}$ microstates and solves the complete $[\mathrm{Xe}] 4 \mathrm{f}^{2}$ and $[\mathrm{Xe}] 4 \mathrm{f}^{1} 5 \mathrm{~d}^{1}$ matrix interactions corresponding to the ground and excited electron configurations of $\operatorname{Pr}^{3+}$, respectively. The atomic spectral terms of the ground [Xe] $4 \mathrm{f}^{2}$ electron configuration are ${ }^{1} \mathrm{~S},{ }^{3} \mathrm{P},{ }^{1} \mathrm{D}$, ${ }^{3} \mathrm{~F},{ }^{1} \mathrm{G},{ }^{3} \mathrm{H}$ and ${ }^{1} \mathrm{I}$, whose total number of microstates equals 91 . The atomic spectral terms of the excited $[\mathrm{Xe}] 4 \mathrm{f}^{1} 5 \mathrm{~d}^{1}$ electron configuration are composed by the low spin ${ }^{1} \mathrm{P},{ }^{1} \mathrm{D},{ }^{1} \mathrm{~F},{ }^{1} \mathrm{G}$ and ${ }^{1} \mathrm{H}$ as well as the high spin ${ }^{3} \mathrm{P},{ }^{3} \mathrm{D},{ }^{3} \mathrm{~F},{ }^{3} \mathrm{G}$ and ${ }^{3} \mathrm{H}$, whose total number of microstates equals 140 . In total, we consider here 231 states. The inter-electron effects are accounted by the SlaterCondon parameters: $F_{2}(\mathrm{ff}), F_{4}(\mathrm{ff})$ and $F_{6}(\mathrm{ff})$ for the $[\mathrm{Xe}] 4 \mathrm{f}^{2}$ configuration and $F_{2}(\mathrm{fd}), F_{4}(\mathrm{fd}), G_{1}(\mathrm{fd}), G_{3}(\mathrm{fd})$ and $G_{5}(\mathrm{fd})$ for the $[\mathrm{Xe}] 4 \mathrm{f}^{1} 5 \mathrm{~d}^{1}$ configuration. ${ }^{52}$ The corresponding values are calculated from the radial functions of the $4 \mathrm{f}$ and $5 \mathrm{~d}$ Kohn-Sham orbitals of $\mathrm{Pr}^{3+}$ (Fig. 2) using eqn (3)-(5). ${ }^{36}$

The spin-orbit coupling constants $\zeta_{4 \mathrm{f}}$ and $\zeta_{5 \mathrm{~d}}$ are estimated by relativistic calculations, as described in the previous section.

The ligand field interaction is expressed on the basis of the merged $4 \mathrm{f}$ and $5 \mathrm{~d}$ atomic orbitals using a twelve by twelve ligand field matrix elements, which can be represented using either the $\mathrm{AOM}^{12,13}$ formalism or the Wybourne-normalized crystal field parameters. ${ }^{53}$ The afore-mentioned parameters are theoretically evaluated by means of the LFDFT and compared with available experimental data listed in Table 2 .

The nephelauxetic effect denominates the reduction of the Slater-Condon parameters from the free ion to the coordinated $\mathrm{Pr}^{3+} \cdot{ }^{14}$ It is characterized by the expansion of the electron cloud toward the ligand atoms shown in Fig. 2. A weak nephelauxetic effect is observed for the 4 f electrons (Fig. 2). Sed contra, the change in the $5 \mathrm{~d}$ radial function from the free ion to $\left(\mathrm{PrF}_{8}\right)^{5-}$ is much more pronounced, leading to a sensible nephelauxetic reduction of the inter-electron parameters. The ligand field part is presented in Table 2 according to the AOM formalism. In the AOM, the ligand field interaction becomes a summation over individual interactions between the $\mathrm{Pr}^{3+}$ and each ligand entity. This interaction possesses a $\sigma, \pi, \delta$ and $\varphi$ bonding nature with the $4 \mathrm{f}$ as well as the $5 \mathrm{~d}$ orbitals (Fig. 3), which is characterized by the $\mathrm{AOM} e_{\sigma}, e_{\pi}, e_{\delta}$ and $e_{\varphi}$ parameters. Nevertheless $e_{\delta}$ and $e_{\varphi}$ are by convenience neglected being engulfed in the $e_{\sigma}$ and $e_{\pi}$ parameters. ${ }^{54}$ In the explicit case of $\left(\mathrm{PrF}_{8}\right)^{5-}$ embedded in $\mathrm{LiYF}_{4}$, we consider two sets of parameters appropriate to each group of four fluoride ligands being symmetrized in $S_{4}$. Thus we obtain $e_{\sigma, 1}(\mathrm{f}), e_{\pi, 1}(\mathrm{f}), e_{\sigma, 2}(\mathrm{f})$ and $e_{\pi, 2}(\mathrm{f})$ perturbation of the $4 \mathrm{f}$; $e_{\sigma, 1}(\mathrm{~d}), e_{\pi, 1}(\mathrm{~d}), e_{\sigma, 2}(\mathrm{~d})$ and $e_{\pi, 2}(\mathrm{~d})$ perturbation of the $5 \mathrm{~d}$; and $e_{\sigma, 1}(\mathrm{fd}), e_{\pi, 1}(\mathrm{fd}), e_{\sigma, 2}(\mathrm{fd})$ and $e_{\pi, 2}(\mathrm{fd})$ perturbation in a second order due to a mixing between the $4 \mathrm{f}$ and the $5 \mathrm{~d}$ orbitals in $S_{4}$ symmetry. The idea to establish the ligand field with respect 
Table 2 Calculated (calc.) and experimental (exp.) Slater-Condon parameters, spin-orbit coupling constants and AOM parameters (in $\mathrm{cm}^{-1}$ ) for the inter-configuration $4 f / 5 d$ interactions in $\left(\mathrm{PrF}_{8}\right)^{5-}$ embedded in $\mathrm{LiYF}_{4}$

\begin{tabular}{llll}
\hline & & $\left(\mathrm{PrF}_{8}\right)^{5-}$ & \\
\cline { 3 - 4 }$\#$ & & Exp. & Calc. \\
\hline 1 & $F_{2}(\mathrm{ff})$ & $306.78^{a}$ & 323.49 \\
2 & $F_{4}(\mathrm{ff})$ & $46.45^{a}$ & 42.24 \\
3 & $F_{6}(\mathrm{ff})$ & $4.53^{a}$ & 4.51 \\
4 & $F_{2}(\mathrm{fd})$ & $216.22^{a}$ & 209.54 \\
5 & $F_{4}(\mathrm{fd})$ & $16.33^{a}$ & 16.35 \\
6 & $G_{1}(\mathrm{fd})$ & $276.49^{a}$ & 294.54 \\
7 & $G_{3}(\mathrm{fd})$ & $26.57^{a}$ & 27.31 \\
8 & $G_{5}(\mathrm{fd})$ & $4.28^{a}$ & 4.36 \\
9 & $\zeta_{4 \mathrm{f}}$ & $750.1^{a}$ & 736.26 \\
10 & $\zeta_{5 \mathrm{~d}}$ & $1149^{a}$ & 944.66 \\
11 & $4_{(\mathrm{fd})}$ & $50966^{a, b}$ & $50943^{b}$ \\
& $\Delta_{\mathrm{AOM}}(\mathrm{fd})$ & - & 24734 \\
12 & $e_{\sigma, 1}(\mathrm{f})$ & $552^{c}$ & 533 \\
13 & $e_{\pi, 1}(\mathrm{f})$ & $103^{c, d}$ & 226 \\
14 & $e_{\sigma, 2}(\mathrm{f})$ & $473^{c, d}$ & 488 \\
15 & $e_{\pi, 2}(\mathrm{f})$ & $88^{c, d}$ & 192 \\
16 & $e_{\sigma, 1}(\mathrm{~d})$ & $12639^{e}$ & 12171 \\
17 & $e_{\pi, 1}(\mathrm{~d})$ & $3482^{e}$ & 2575 \\
18 & $e_{\sigma, 2}(\mathrm{~d})$ & $10512^{e}$ & 10078 \\
19 & $e_{\pi, 2}(\mathrm{~d})$ & $4875^{e}$ & 3314 \\
20 & $e_{\sigma, 1}(\mathrm{fd})$ & - & 1864 \\
21 & $e_{\pi, 1}(\mathrm{fd})$ & - & 850 \\
22 & $e_{\sigma, 2}(\mathrm{fd})$ & - & 1534 \\
23 & $e_{\pi, 2}(\mathrm{fd})$ & - & 244 \\
& & &
\end{tabular}

${ }^{a}$ These parameters are taken from ref. $55 .{ }^{b}$ The $\Delta(\mathrm{fd})$ represents the difference of the barycentres of the energy between the multiplet levels from the $[\mathrm{Xe}] 4 \mathrm{f}^{1} 5 \mathrm{~d}^{1}$ and $[\mathrm{Xe}]^{4} \mathrm{f}^{2}$ configuration of $\mathrm{Pr}^{3+}$. It is listed for clarity being replaced by the $\Delta_{\mathrm{AOM}}(\mathrm{fd}) .{ }^{54}{ }^{c}$ We refer to the parameters given for the optical measurement reported in ref. $40 .{ }^{d}$ We refer to ref. 40 and use the ratios $e_{\sigma}(\mathrm{f}) / e_{\pi}(\mathrm{f})=5.34, e_{\sigma, 1}(\mathrm{f}) / e_{\sigma, 2}(\mathrm{f})=\left(d_{1} / d_{2}\right)^{7}$ and $e_{\pi, 1}(\mathrm{f}) / e_{\pi, 2}(\mathrm{f})=\left(d_{1} / d_{2}\right)^{7}$ given there. ${ }^{e}$ We consider the Wybourne parameters in ref. 55 and transform them to AOM using the coordinates of $\left(\mathrm{PrF}_{8}\right)^{5-}$ in Table 1.

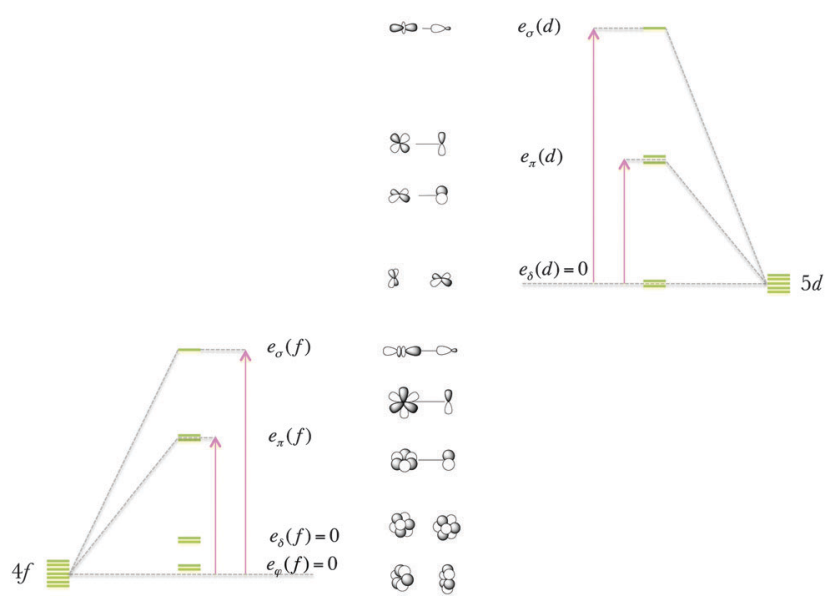

Fig. 3 Graphical representation of the 4f-type (left-hand-side) and 5d-type (right-hand-side) AOM parameters.

to the AOM results is the fact that the AOM are intuitive parameters, which can be transferred to comparable systems. Recently we constructed the extension of the AOM for two-openshell $\mathrm{f}$ and d electrons. ${ }^{54}$ Because the AOM matrices are not nontraceless, as usual ligand field parameterization, it is convenient to redefine a new energy gap $\Delta_{\mathrm{AOM}}(\mathrm{fd})$ instead of $\Delta(\mathrm{fd}),{ }^{54}$ which for clarity is also listed in Table 2. The ligand field Hamiltonian (eqn (1)) operates in total with twenty-three parameters (Table 2). The computed parameters are compared to experimental measurements, due to the previous work of Urland ${ }^{40}$ and Reid et al., ${ }^{55}$ who have used parameters to fit their experimental spectra of $\mathrm{LiYF}_{4}: \mathrm{Pr}^{3+}$. Note however that experimental values for the mixed 4f/5d-type AOM parameters are not provided, since they are not found in the literature. The calculated parameters are in general of the order of magnitude of the experimental values (Table 2), apart from discrepancies originating from the selfconsistent error in the DFT setting.

More explicitly, the $F_{2}(\mathrm{ff})$ and $G_{1}(\mathrm{fd})$ parameters are overestimated if compared to the experimental values, while the $F_{4}(\mathrm{ff})$ and the $F_{2}(\mathrm{fd})$ are underestimated, aspects that are already visible in earlier investigations. ${ }^{8,39,54}$ The 4 f-type AOM parameters are particularly overestimated in the $\pi$-interaction (Table 2 ), depending on the choice of the exchange and correlation functional in the DFT setting. This does underpin the motivation of using a hybrid functional along the LFDFT ${ }^{8}$ although the pure functional performed better in the geometry optimization. Table 3 shows the multiplet energy levels corresponding to the ${ }^{3} \mathrm{H}$ ground state of the ground [Xe] $4 \mathrm{f}^{2}$ electron configuration of $\mathrm{Pr}^{3+}$ in $\mathrm{LiYF}_{4}: \mathrm{Pr}^{3+}$. Under the influence of the spin-orbit coupling, the ${ }^{3} \mathrm{H}$ splits over ${ }^{3} \mathrm{H}_{4},{ }^{3} \mathrm{H}_{5}$ and ${ }^{3} \mathrm{H}_{6}$ terms, whose total number of microstates equals 9, 11 and 13, respectively. These terms are then split into ligand field components according to $a, b$ and $e$ irreducible representations (irreps) of the $S_{4}$ point group (Table 3). A good qualitative agreement between the experimentally observed energy levels ${ }^{56}$ and the non-empirical calculated ones is observed (Table 3). The deviation is evaluated, in least mean squares, about $17 \mathrm{~cm}^{-1}$, which is mostly due to the misrepresentation of the $e_{\pi, 1}(\mathrm{f})$ and $e_{\pi, 2}(\mathrm{f})$ parameters by the non-empirical calculation. On the other hand, the calculation underestimates the 5 d-type AOM parameters in the $\pi$-interaction (Table 2 ). The ligand field splitting of the $5 \mathrm{~d}$ orbitals, which confers the most important interaction within the $[\mathrm{Xe}] 4 \mathrm{f}^{1} 5 \mathrm{~d}^{1}$ configuration of $\mathrm{Pr}^{3+}$, deserves a special attention. In $\mathrm{LiYF}_{4}: \mathrm{Pr}^{3+}$, the splitting pattern of the five-fold $5 \mathrm{~d}$ orbitals of $\operatorname{Pr}^{3+}$ follows the representations $b, a, e$ and $b$ of the $S_{4}$ point group. Following the Wybourne formalism, ${ }^{53}$ three parameters are taken into consideration such as $B_{0}^{2}(\mathrm{~d}), B_{0}^{4}(\mathrm{~d})$ and $B_{4}^{4}(\mathrm{~d})$ since the $B_{-4}^{4}(\mathrm{~d})$ is the complex conjugate of the analogue $B_{4}^{4}(\mathrm{~d})$. The first two parameters are by definition real, whereas the third one is complex. It is possible to retrieve a real value of $B_{4}^{4}(\mathrm{~d})$ by forcing the cluster to belong to the higher $D_{2 \mathrm{~d}}$ point group, as it is already observed in other studies. ${ }^{9,55,57}$ Taking the AOM (Table 2), we calculate (in $\mathrm{cm}^{-1}$ ) 3256, -23626 and $-23576+i 13410$, respectively for $B_{0}^{2}(\mathrm{~d}), B_{0}^{4}(\mathrm{~d})$ and $B_{4}^{4}(\mathrm{~d})$. The diagonalization of the ligand field potential $\left(V_{\mathrm{LF}}\right)$ yields the eigenvalues and the associated normalized eigenvectors (Table 4). The coefficients which appear in these eigenvectors are complex numbers specific to the basis of the one-electron ligand field matrix $\left|l, m_{l}\right\rangle$, i.e. the merged $4 \mathrm{f}(l=3)$ and $5 \mathrm{~d}(l=2)$ atomic orbitals. In the explicit case of the $5 \mathrm{~d}$ ligand field, the results are numerically given in Table 4 , represented with respect to the Wybourne formalism. We use the indices ( $\xi$ and $\eta$ ) to 
Table 3 Calculated (calc.) and experimental (exp.) multiplet energy levels (in $\mathrm{cm}^{-1}$ ) corresponding to the spectral term ${ }^{3} \mathrm{H}$ ground state of the $[\mathrm{Xe}] 4 \mathrm{f}^{2}$ configuration of $\mathrm{Pr}^{3+}$ in $\left(\mathrm{PrF}_{8}\right)^{5-}$ embedded in LiYF 4 . The energy of the first $B$ level of the ${ }^{3} \mathrm{H}_{4}$ term is taken as the origin of the energy

\begin{tabular}{llllr}
\hline & $\left(\mathrm{PrF}_{8}\right)^{5-}$ & & & \\
\cline { 2 - 5 }$\#$ & Terms & Level & Exp. $^{a}$ & Calc. \\
\hline 1 & ${ }^{3} \mathrm{H}_{4}$ & $\mathrm{~B}$ & 0 & 0 \\
2,3 & & $\mathrm{E}$ & 79 & 75 \\
4 & & $\mathrm{~A}$ & 220 & 392 \\
5 & & $\mathrm{~A}$ & - & 420 \\
6 & $\mathrm{~A}$ & - & 467 \\
7,8 & $\mathrm{E}$ & 496 & 491 \\
9 & & $\mathrm{~B}$ & - & 503 \\
10,11 & $\mathrm{E}$ & 2272 & 2255 \\
12 & $\mathrm{~A}$ & 2253 & 2264 \\
13 & ${ }^{3} \mathrm{H}_{5}$ & $\mathrm{~B}$ & 2280 & 2265 \\
14 & & $\mathrm{~A}$ & 2297 & 2331 \\
15,16 & $\mathrm{E}$ & 2341 & 2490 \\
17 & & $\mathrm{~B}$ & 2549 & 2541 \\
18 & & $\mathrm{~A}$ & - & 2578 \\
19,20 & $\mathrm{E}$ & - & 2579 \\
21 & & $\mathrm{~B}$ & 4314 & 4331 \\
22 & & $\mathrm{~A}$ & - & 4361 \\
23,24 & $\mathrm{E}$ & 4394 & 4369 \\
25 & ${ }^{3} \mathrm{H}_{6}$ & $\mathrm{~B}$ & - & 4441 \\
26,27 & & $\mathrm{E}$ & 4454 & 4470 \\
28 & & $\mathrm{~A}$ & 4486 & 4665 \\
29 & & B & 4557 & 4678 \\
30 & & $\mathrm{~A}$ & - & 4818 \\
31,32 & $\mathrm{E}$ & 4907 & 4828 \\
33 & & $\mathrm{~B}$ & 4945 & 4853 \\
${ }^{a}$ Taken from & ref. 56. & & & \\
\hline
\end{tabular}

differentiate between the components of the two-fold degenerate eigenvalues of $e$ symmetry and ( $\varepsilon$ and $\zeta$ ) between the two distinct eigenvalues belonging to the same $b$ irrep. For these latter ones, the eigenvectors in case of enforcement to $D_{2 \mathrm{~d}}$ coordination of $\mathrm{Pr}^{3+}$ can be determined analytically by symmetry adapted linear combination of the $|2,+2\rangle$ and the $|2,-2\rangle$ basis functions within a symmetrical (eqn (6)) and anti-symmetrical (eqn (7)) composition. Note however that the expressions in eqn (6) and (7) are no longer retrieved in the actual $S_{4}$ coordination of $\operatorname{Pr}^{3+}$ (Table 4 ) and help for a better visibility of the eigenvectors given in Table 4 .

$$
\begin{aligned}
& \left|2, b_{\varepsilon}\right\rangle=\frac{\sqrt{2}}{2}(|2,-2\rangle+|2,+2\rangle) \\
& \left|2, b_{\zeta}\right\rangle=\frac{\sqrt{2}}{2}(|2,-2\rangle-|2,+2\rangle)
\end{aligned}
$$

The eigenvectors (Table 4) contain small implications of the 4 f orbitals caused by the $4 \mathrm{f} / 5 \mathrm{~d}$-type AOM parameters (Table 2), which have to be taken into account in the calculation for a realistic simulation of the electronic structure of $\left(\mathrm{PrF}_{8}\right)^{5-}$ embedded in $\mathrm{LiYF}_{4}$. The splitting of the energy of the $5 \mathrm{~d}$ orbitals (Table 4) is in line with what can be deduced from experiment. ${ }^{55}$

Addressing a trustable simulation of the $\mathrm{f}-\mathrm{d}$ transitions in lanthanide compounds is a challenging problem in computational chemical science. Ligand field theory operated along the non-empirical DFT offers here a promising approach, since a perfect match between the non-empirical parameters and the experimental results might be experienced. There is another aspect making the theoretical consideration very important, when characterizing experimentally observed excitation and emission spectra. An empirical fit to the experimental spectrum with respect to the ligand field theory requires in low symmetry cases a large number of independent parameters. ${ }^{58}$ The treatment of the present $S_{4}$ coordination of the $\operatorname{Pr}^{3+}$ ion is still possible: twenty-three parameters (Table 2). However, in the case of no symmetry, the experimental fit is not affordable, having a total of ninety free parameters. In such a situation the computational counterpart offers valuable guidelines in contouring the relative values of the parametric scheme. We use the calculated data from Table 2 to parameterize the ligand field Hamiltonian (eqn (1)), the eigenvalues of which relate the multiplet energy levels arising from $[\mathrm{Xe}] 4 \mathrm{f}^{2}$ and the $[\mathrm{Xe}] 4 \mathrm{f}^{1} 5 \mathrm{~d}^{1}$ configurations of $\mathrm{Pr}^{3+}$ in $\mathrm{LiYF}_{4}: \mathrm{Pr}^{3+}$. These eigenvalues are graphically represented in Fig. 4 using different colours. The corresponding numerical values are given in the ESI. $\dagger$ The excitation $[\mathrm{Xe}] 4 \mathrm{f}^{2} \rightarrow[\mathrm{Xe}] 4 \mathrm{f}^{1} 5 \mathrm{~d}^{1}$ and the emission $[\mathrm{Xe}] 4 \mathrm{f}^{1} 5 \mathrm{~d}^{1} \rightarrow[\mathrm{Xe}] 4 \mathrm{f}^{2}$ are allowed electric dipole transitions. The matrix elements of the electric dipole moment operator are determined using the vector coupling basis, ${ }^{52}$ from which the radiative transition probability based on zero-phonon lines (Fig. 4, in black) is determined as proportional to the $4 \mathrm{f}-5 \mathrm{~d}$ mixing. From Fig. 4 , the [Xe] $4 \mathrm{f}^{1} 5 \mathrm{~d}^{1}$ configuration is characterized by three dominant bands, in line with the experimental excitation spectrum given by Reid et al. ${ }^{55}$

This does not directly reflect the splitting of the $5 \mathrm{~d}$ orbitals shown in Table 4, where four bands would be expected, because the fourth band is less resolved due to the small energy difference calculated between the double degenerate $|2, e\rangle$ and the $\left|2, b_{\zeta}\right\rangle$ states (Table 4 ). In a strict numerical simulation, we can tune the influence of all the parameters in Table 2 by

Table 4 Calculated (calc.) eigenvalues (in $\mathrm{cm}^{-1}$ ) of the ligand field potential in the $5 \mathrm{~d}$ orbitals of $\mathrm{Pr}^{3+}$ in $\mathrm{LiYF}_{4}$ : $\mathrm{Pr}^{3+}$ together with the corresponding eigenvectors

\begin{tabular}{lll}
\hline & $\left(\mathrm{PrF}_{8}\right)^{5-}$ & \\
\cline { 2 - 3 } & Calc. & Eigenvectors \\
\hline$\left|2, b_{\varepsilon}\right\rangle$ & -12626 & $(0.6147-i 0.3496)|2,-2\rangle+0.7071|2,+2\rangle-(0.0019+i 0.0005)|3,0\rangle$ \\
$|2, a\rangle$ & -6072 & $0.9990|2,0\rangle+(0.0091+i 0.0306)|3,-2\rangle+(0.0091-i 0.0306)|3,+2\rangle$ \\
$\left|2, e_{\eta}\right\rangle$ & 4850 & $0.7152|2,-1\rangle+(0.4704-i 0.5161)|2,+1\rangle-(0.0070+i 0.0115)|3,-3\rangle-(0.0135+i 0.0026)|3,+3\rangle$ \\
$\left|2, e_{\xi}\right\rangle$ & 4850 & $+(0.0141-i 0.0053)|3,-1\rangle+(0.0131-i 0.0067)|3,+1\rangle$ \\
$\left|2, b_{\zeta}\right\rangle$ & 8997 & $0.7107|2,-1\rangle-(0.4639-i 0.5281)|2,+1\rangle+(0.0068-i 0.0117)|3,-3\rangle-(0.0134+i 0.026)|3,+3\rangle$ \\
& & $+(0.0140-i 0.0053)|3,-1\rangle-(0.0131-i 0.0070)|3,+1\rangle$ \\
& & $0.7067|2,-2\rangle-(0.6142+i 0.3494)|2,+2\rangle+(0.0090-i 0.0341)|3,0\rangle$
\end{tabular}




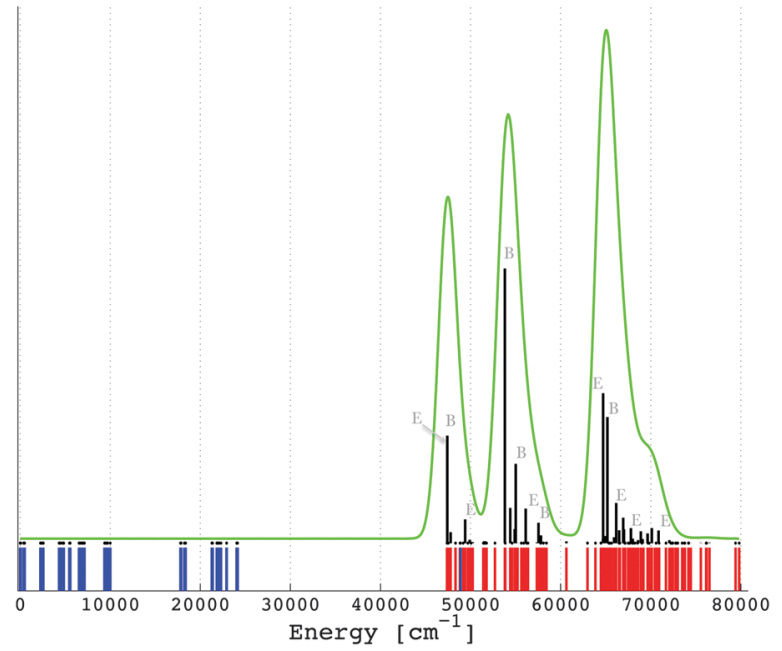

Fig. 4 Calculated multiplet energy levels from the $[\mathrm{Xe}] 4 \mathrm{f}^{2}$ (in blue) and $[\mathrm{Xe}] 4 f^{1} 5 d^{1}$ (in red) configurations of $\mathrm{Pr}^{3+}$ in $\mathrm{LiYF}_{4}: \mathrm{Pr}^{3+}$ (see also the ESI $\dagger$ ) together with the intensities of the excitation $[\mathrm{Xe}] 4 \mathrm{f}^{2} \rightarrow[\mathrm{Xe}] 4 \mathrm{f}^{1} 5 \mathrm{~d}^{1}$ transitions, i.e. zero-phonon lines (in black). The green curve represents a superimposition of a Gaussian band with a width of $1000 \mathrm{~cm}^{-1}$ on the zero-phonon lines.

setting some values of them to zero and seeing the change in Fig. 4. We find out that the excitation transitions (Fig. 4) are not only due to the ligand field splitting of the $5 \mathrm{~d}$ orbitals but also to a subtle influence of the spin-orbit coupling in the $4 \mathrm{f}$ electrons. On the other hand the spin-orbit coupling of the $5 \mathrm{~d}$ electrons and the ligand field of the $4 \mathrm{f}$ orbitals have a weak effect.

Besides the general lighting applications, the multiplet energy levels of $\mathrm{Pr}^{3+}$ a priori favour a quantum cutting process by possible photon cascade emissions from the $[\mathrm{Xe}] 4 \mathrm{f}^{1} 5 \mathrm{~d}^{1}$ to the $[\mathrm{Xe}] 4 \mathrm{f}^{2}$ levels. $^{3-5}$ The quantum cutter exhibits a two or more photon luminescence process, where at least two low energy photons are emitted per one high energy photon absorbed. ${ }^{3-5}$ Therefore, quantum efficiencies larger than $100 \%$ are achievable, which was already demonstrated in the literature. ${ }^{3-5,59,60}$ This optical manifestation is possible because of the energy gap between the $[\mathrm{Xe}] 4 \mathrm{f}^{2}$ and the $[\mathrm{Xe}] 4 \mathrm{f}^{1} 5 \mathrm{~d}^{1}$ multiplets. $\operatorname{Pr}^{3+}$ doped into fluoride hosts mostly achieves such a phenomenon. A systematic review of the quantum cutting process in general is recently available. ${ }^{61}$ Unfortunately in the present $\mathrm{LiYF}_{4}: \operatorname{Pr}^{3+}$ case, there is no energy gap observed between the $[\mathrm{Xe}] 4 \mathrm{f}^{2}$ (Fig. 4 , in blue) and the [Xe] $4 \mathrm{f}^{1} 5 \mathrm{~d}^{1}$ (Fig. 4 , in red) manifolds, i.e. the multiplet levels arising from the $[\mathrm{Xe}] 4 \mathrm{f}^{1} 5 \mathrm{~d}^{1}$ configuration overlap those from the $[\mathrm{Xe}] 4 \mathrm{f}^{2}$ (see Fig. 4). Therefore, the $\mathrm{LiYF}_{4}: \mathrm{Pr}^{3+}$ system is not a quantum cutter, in agreement with the earlier experimental observations, ${ }^{62}$ where the observed emission spectrum is dominated by the inter-configuration $[\mathrm{Xe}] 4 \mathrm{f}^{1} 5 \mathrm{~d}^{1} \rightarrow[\mathrm{Xe}] 4 \mathrm{f}^{2}$ transitions in the spectral range of $200 \mathrm{~nm}$ and $400 \mathrm{~nm}$. A simulation of the emission spectrum is possible, taking advantage of the dipole allowed $\mathrm{f}-\mathrm{d}$ transitions. This simulation of the emission $[\mathrm{Xe}] 4 \mathrm{f}^{1} 5 \mathrm{~d}^{1} \rightarrow[\mathrm{Xe}] 4 \mathrm{f}^{2}$ is done by taking into account a change of the geometry of $\left(\mathrm{PrF}_{8}\right)^{5-}$ embedded in $\mathrm{LiYF}_{4}$ in the excited configuration. Although apparently counter-intuitive, in the excited $[\mathrm{Xe}] 4 \mathrm{f}^{1} 5 \mathrm{~d}^{1}$ state, the coordination bond lengths and implicitly the overall ligand field strength are higher than in the
$[\mathrm{Xe}] 4 \mathrm{f}^{2}$ ground configuration. We encountered and discussed such a situation in our precedent work ${ }^{35}$ analyzing the octahedral cluster $\left(\mathrm{CeCl}_{6}\right)^{3-}$. A similar behavior is expected in the actual $\mathrm{Pr}^{3+}$ system. The situation is due to the fact that the bonding regime in lanthanide complexes is mainly ensured by the $5 \mathrm{~d}$ orbitals, ${ }^{63,64}$ which behave as acceptors for the lone pairs of the ligands, while the $4 \mathrm{f}$ shell is too shielded to play an effective role (outside the pure electrostatics of the whole lanthanide body). The promotion of one electron in the $5 \mathrm{~d}$ virtuals enhances their bonding capability, by an increment in the nephelauxetic effect, favorable to the overlap with the environment. Consequently, a surge of electron population of the empty $5 \mathrm{~d}$ orbitals results in shortening of the lanthanide-ligand bond lengths. Considering again the DFT geometry optimization based on the molecular cluster approach (Methodology section), we work with the excited $[\mathrm{Xe}] 4 \mathrm{f}^{1} 5 \mathrm{~d}^{1}$ configuration of $\mathrm{Pr}^{3+}$ in the system $\left(\mathrm{PrF}_{8}\right)^{5-}$ embedded in $\mathrm{LiYF}_{4}$. In this respect, the optimized structure of $\left(\mathrm{PrF}_{8}\right)^{5-}$ in Table 1 is taken, then the electron population of the orbitals is changed inasmuch as one electron is evenly distributed amongst the seven-fold $4 \mathrm{f}$ orbitals and the other one is placed in the lowest energy $\left|2, b_{\varepsilon}\right\rangle$ component of the $5 \mathrm{~d}$ orbitals (Table 4 ). We let the structure to relax following the totally symmetric displacements of $\left(\mathrm{PrF}_{8}\right)^{5-}$ in its $S_{4}$ coordination, according to the procedure described in the methodology section within the constrained electronic structure. We obtain by means of the VWN functional the following coordinates using the same representation as Table 1:

$$
\begin{aligned}
d_{1} & =2.2881 \AA, \theta_{1}=66.33^{\circ} \text { and } \phi_{1}=-37.63^{\circ} ; \\
d_{2} & =2.3394 \AA, \theta_{2}=138.09^{\circ} \text { and } \phi_{2}=-35.05^{\circ},
\end{aligned}
$$

where noticeable shortening of the Pr-F bond lengths is observed corroborating the earlier account for the $\mathrm{Ce}^{3+}$ system. ${ }^{35}$ The structural changes of the $\left(\mathrm{PrF}_{8}\right)^{5-}$ in the excited configuration of $\mathrm{Pr}^{3+}$ are important features for the model of any $\mathrm{f}-\mathrm{d}$ emission lines. Experimentally, it is characterized by the Stokes shift, which is in general accompanying the lanthanide luminescence. ${ }^{65,66}$ Shorter bond lengths correspond to larger ligand field splitting for both $4 \mathrm{f}$ and $5 \mathrm{~d}$ orbitals, which for the latter one is particularly significant, as obtained from the LFDFT. The AOM parameters in Table 2 become (in $\left.\mathrm{cm}^{-1}\right) e_{\sigma, 1}(\mathrm{~d})=13309, e_{\pi, 1}(\mathrm{~d})=2219, e_{\sigma, 2}(\mathrm{~d})=$ 10226 and $e_{\pi, 2}(\mathrm{~d})=3971$, leading to an enlargement of the gap between higher and lower values of the 5d-type ligand field eigenvalues. This is seen when comparing excited state values of the ligand field potential (in $\mathrm{cm}^{-1}$ ): $-14080,-5960,4832$ and 10376 , respectively for the $\left|2, b_{\varepsilon}\right\rangle,|2, a\rangle,|2, e\rangle$ and $\left|2, b_{\zeta}\right\rangle$ levels with the quantities from Table 4 . The 5d-type AOM parameters in the excited-state case are slightly but firmly higher than the groundstate ones, except the case of the $e_{\pi, 1}(\mathrm{~d})$ value which shows a reverse change. Since in this case the used four parameters are uniquely determined from the four energy spacings of the relative 5d-type ligand field scheme, the effect is not a numerical ambiguity, being however difficult to understand. Tentatively, we can assign the slight lapse to a general drawback of the ligand field schemes in surroundings without the inversion center, namely the so-called holohedrization effect. ${ }^{67}$ In this conjuncture, a certain artificial compensation may appear between $e_{\pi, 1}(\mathrm{~d})$ and 


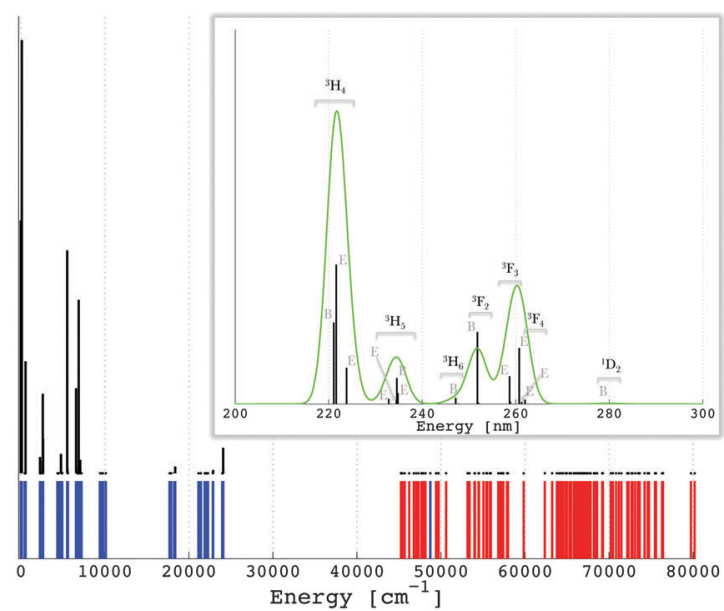

Fig. 5 Calculated multiplet energy levels from the $[X e] 4 f^{2}$ (in blue) and $[\mathrm{Xe}] 4 f^{1} 5 d^{1}$ (in red) configurations of $\mathrm{Pr}^{3+}$ in $\mathrm{LiYF}_{4}: \mathrm{Pr}^{3+}$ (see also the ESI $\dagger$ ) together with the intensities of the emission $[\mathrm{Xe}] 4 \mathrm{f}^{1} 5 d^{1} \rightarrow[\mathrm{Xe}] 4 \mathrm{f}^{2}$ transitions, i.e. zero-phonon lines (in black). The energy region showing the maximum emission transitions is magnified and represented in units of wavelength $(\mathrm{nm})$. The green curve represents a superimposition of a Gaussian band with a width of $2 \mathrm{~nm}$ on the emission lines.

$e_{\pi, 2}(\mathrm{~d})$ values. However, we will not advance here to the further discrimination of possible subtle parameterization issues. Since the whole ligand field strength goes towards the noticed intensification in the excited-state, the particular situation of the $e_{\pi, 1}(\mathrm{~d})$ value does not impinge upon the general conclusion about enhanced covalency along with the promotion of one electron in the 5d-type virtuals of the lanthanide coordination spheres. The other parameters (Table 2) are less influenced, as expected and proved by the LFDFT calculation (see also the ESI $\dagger$ ). The emission lines originating from the lowest $[\mathrm{Xe}] 4 \mathrm{f}^{1} 5 \mathrm{~d}^{1}$ state of $\mathrm{Pr}^{3+}$ in $\mathrm{LiYF}_{4}: \mathrm{Pr}^{3+}$ are given in Fig. 5. In line with the experimental observation, ${ }^{62}$ the inter-configuration $[\mathrm{Xe}] 4 \mathrm{f}^{1} 5 \mathrm{~d}^{1} \rightarrow[\mathrm{Xe}] 4 \mathrm{f}^{2}$ transitions are mainly in the ultraviolet spectral range, where the most intense is $[\mathrm{Xe}] 4 \mathrm{f}^{1} 5 \mathrm{~d}^{1} \rightarrow[\mathrm{Xe}] 4 \mathrm{f}^{2}\left({ }^{3} \mathrm{H}\right)$ (Table 3).

\section{Conclusions}

In the beginning of the twenty first century, many governments inclined towards the phase-out of the incandescent light bulbs, which have lightened the world for over 100 years since their first development by Edison. This corresponds to the effort to diminish nowadays considerable energy demand. Therefore the progress toward modern domestic lighting will focus in the future on how efficiently the energy will be consumed. Light emitting diode (LED) bulbs are amongst the light source alternatives and offer a bunch of advantages, due to the interesting optical properties exhibited by some lanthanide phosphors.

In the perspective that the theory can help the experiment in finding the optimal materials by identifying clue parameters on a first principle route, we have drawn some points on this line of the structure-property correlations, potentially serving material engineering. The model relevant for the calculation of the optical properties of lanthanide compounds is based on the phenomenological Hamiltonian adjusted from the ligand field theory. It is demonstrated that using standard quantum chemistry tools, e.g. Density Functional Theory (DFT), the parameterization of the ligand field effective Hamiltonian can be obtained in a very efficient way. The obtained parameters are in principle transferable to other systems of similar nature, further investigation being necessary for the complete charting of all the lanthanide-ligand couples acting as potential candidates for phosphor materials.

In this work, we have determined by means of DFT calculations the multiplet energy levels arising from the ground $[\mathrm{Xe}] 4 \mathrm{f}^{2}$ and excited [Xe] $4 \mathrm{f}^{1} 5 \mathrm{~d}^{1}$ electron configurations of $\mathrm{Pr}^{3+}$ in the two-openshell problem encountered in the system $\mathrm{LiYF}_{4}: \mathrm{Pr}^{3+}$. The treatment of the local distortions due to the presence of the $\mathrm{Pr}^{3+}$ impurity in $\mathrm{LiYF}_{4}$ is addressed by standard geometry optimization, circumventing the use of band structure algorithms and gaining aspects demanded by a pure chemical vision like the geometry in the excited states. The parametric scheme offered by ligand field theory is used to provide a chemical intuitiveness of the nonempirical investigation. The calculated parameters are therefore compared to available experimental results collected also here for the purpose of the work. A qualitative agreement between the nonempirical investigation and the experimental findings is stated, allowing the prediction of the luminescence of $\mathrm{LiYF}_{4}: \mathrm{Pr}^{3+}$ in the frame of $4 \mathrm{f}$ and $5 \mathrm{~d}$ electrons. The experimentally observed excitation and emission spectra are theoretically reproduced here, enabling a better understanding of the optical phenomenon and a good connection between spectroscopy studies and theoretical investigations in inorganic chemical science.

\section{Acknowledgements}

This work is supported by the Swiss National Science Foundation (SNF) and the Swiss State Secretariat for Innovation and Research. Support from the UEFISCDI Romania research grant PCE 14/2013 is also acknowledged.

\section{Notes and references}

1 S. Nakamura and G. Fasol, The Blue Laser Diode, Springer, Berlin, 1997.

2 T. Jüstel, H. Nikol and C. Ronda, Angew. Chem., Int. Ed., 1998, 37, 3084.

3 J. L. Sommerdijk, A. Bril and A. W. de Jager, J. Lumin., 1974, 8, 341.

4 W. W. Piper, J. A. DeLuca and F. S. Ham, J. Lumin., 1974, $8,344$.

5 R. T. Wegh, H. Donker, K. D. Oskam and A. Meijerink, Science, 1999, 29, 663.

6 A. Richter, E. Heumann, E. Osiac, G. Huber, W. Seelert and A. Diening, Opt. Lett., 2004, 29, 2638.

7 T. Gün, P. Metz and G. Huber, Appl. Phys. Lett., 2011, 99, 181103.

8 H. Ramanantoanina, W. Urland, F. Cimpoesu and C. Daul, Phys. Chem. Chem. Phys., 2013, 15, 13902. 
9 M. D. Faucher and O. K. Moune, Phys. Rev. A: At., Mol., Opt. Phys., 1997, 55, 4150.

10 M. Krosnicki, A. Kedziorski, L. Seijo and Z. Barandiaran, J. Phys. Chem. A, 2014, 118, 358.

11 P. A. Tanner, C. S. K. Mak and M. D. Faucher, J. Chem. Phys., 2001, 114, 10860.

12 C. Schäffer and C. Jørgensen, Mol. Phys., 1965, 9, 401.

13 W. Urland, Chem. Phys., 1976, 14, 393.

14 P. A. Tanner and Y. Y. Yeung, J. Phys. Chem. A, 2013, 117, 10726.

15 G. te Velde, F. M. Bickelhaupt, S. J. A. van Gisbergen, C. F. Guerra, E. J. Baerends, J. G. Snijders and T. Ziegler, J. Comput. Chem., 2001, 22, 931.

16 C. F. Guerra, J. G. Snijders, G. te Velde and E. J. Baerends, Theor. Chem. Acc., 1998, 99, 391.

17 E. J. Baerends, T. Ziegler, J. Autschbach, D. Bashford, A. Berces, F. M. Bickelhaupt, C. Bo, P. M. Boerrigter, L. Cavallo, D. P. Chong, L. Deng, R. M. Dickson, D. E. Ellis, M. van Faassen, L. Fan, T. H. Fischer, C. F. Guerra, A. Ghysels, A. Giammona, S. J. A. van Gisbergen, A. W. Götz, J. A. Groeneveld, O. V. Gritsenko, M. Grüning, S. Gusarov, F. E. Harris, P. van den Hoek, C. R. Jacob, H. Jacobsen, L. Jensen, J. W. Kaminski, G. van Kessel, F. Koostra, A. Kovalenko, M. V. Krykunov, E. van Lenthe, D. A. McCormack, A. Michalak, M. Mitoraj, J. Neugebauer, V. P. Nicu, L. Noodleman, V. P. Osinga, S. Patchkovskii, P. H. T. Philipsen, D. Post, C. C. Pye, W. Ravenek, J. I. Rodriguez, P. Ros, P. R. T. Shipper, G. Schreckenbach, J. S. Seldenthuis, M. Seth, J. G. Snijders, M. Sola, M. Swart, D. Swerhone, G. te Velde, P. Vernooijs, L. Versluis, L. Vissher, O. Visser, F. Wang, T. Wesolowski, E. M. van Wezenbeek, G. Wiesenekker, S. K. Wolff, T. K. Woo and A. L. Yarkolev, ADF2013.01, available at http://www.scm.com.

18 J. S. Griffith, The Theory of Transition Metal Ions, Cambridge University Press, Cambridge, 1961.

19 B. N. Figgis and M. A. Hitchman, Ligand Field Theory and its Applications, Wiley-VCH, New York, 2000.

20 B. R. McGarvey and J. Telser, Inorg. Chem., 2012, 51, 6000.

21 M. Atanasov, P. Surawatanawong, K. Wieghardt and F. Neese, Coord. Chem. Rev., 2013, 257, 27.

22 F. Cimpoesu, N. Dragoe, H. Ramanantoanina, W. Urland and C. Daul, Phys. Chem. Chem. Phys., 2014, 16, 11337.

23 J. Paulovic, F. Cimpoesu, M. Ferbinteanu and K. Hirao, J. Am. Chem. Soc., 2004, 126, 3321.

24 M. Ferbinteanu, T. Kajiwara, K.-Y. Choi, H. Nojiri, A. Nakamoto, N. Kojima, F. Cimpoesu, Y. Fujimura, S. Takaishi and M. Yamashita, J. Am. Chem. Soc., 2006, 128, 9008.

25 S. Tanase, M. Ferbinteanu and F. Cimpoesu, Inorg. Chem., 2011, 50, 9678.

26 F. Cimpoesu, F. Dahan, S. Ladeira, M. Ferbinteanu and J.-P. Costes, Inorg. Chem., 2012, 51, 11279.

27 M. Atanasov, C. A. Daul and C. Rauzy, Struct. Bonding, 2004, 106, 97.

28 D. Gatteschi and R. Sessoli, Angew. Chem., Int. Ed., 2003, 42, 268.
29 M. F. Reid, F. S. Richardson and P. A. Tanner, Mol. Phys., 1987, 60, 881.

30 L. Noodleman and E. R. Davidson, Chem. Phys., 1986, 109, 131.

31 E. Ruiz, P. Alemany, S. Alvarez and J. Cano, J. Am. Chem. Soc., 1997, 119, 1297.

32 M. Mitani, K. Mori, Y. Takano, D. Yamaki, Y. Yoshioka and K. Yamaguchi, Chem. Phys., 2000, 113, 4035.

33 E. K. U. Gross, L. N. Oliveria and W. Kohn, Phys. Rev. A: At., Mol., Opt. Phys., 1988, 37, 2809.

34 E. Garcia and R. R. Ryan, Acta Crystallogr., Sect. C: Cryst. Struct. Commun., 1993, 49, 2053.

35 H. Ramanantoanina, W. Urland, A. Garcia-Fuente, F. Cimpoesu and C. Daul, Chem. Phys. Lett., 2013, 588, 260.

36 H. Ramanantoanina, W. Urland, A. Garcia-Fuente, F. Cimpoesu and C. Daul, Phys. Chem. Chem. Phys., 2014, 16, 14625.

37 I. B. Bersuker, The Jahn-Teller Effect, Cambridge University Press, Cambridge, 2006.

38 I. B. Bersuker and V. Z. Polinger, Vibronic interactions in Molecules and Crystals, Springer-Verlag, Berlin, 1989.

39 W. W. Parson, Modern Optical Spectroscopy, Springer-Verlag, Berlin, 2007.

40 W. Urland, Chem. Phys. Lett., 1981, 77, 58.

41 Vishwamittar and S. P. Puri, J. Phys. C: Solid State Phys., 1974, 7, 1337.

42 J. C. Slater, Qunatum Theory of Molecules and Solids, McGraw-Hill, NewYork, 1963.

43 S. H. Vosko, L. Wilk and M. Nusair, Can. J. Phys., 1980, 58, 1200.

44 A. D. Becke, Phys. Rev. A: At., Mol., Opt. Phys., 1988, 38, 3098.

45 J. P. Perdew, Phys. Rev. B: Condens. Matter Mater. Phys., 1986, 33, 8822 .

46 J. P. Perdew, J. A. Chevary, S. H. Vosko, K. A. Jackson, M. R. Pederson, D. J. Sing and C. Fiolhais, Phys. Rev. B: Condens. Matter Mater. Phys., 1992, 46, 6671.

47 M. T. Yin and M. L. Cohen, Phys. Rev. Lett., 1980, 45, 1004.

48 C. Daul, Int. J. Quantum Chem., 1994, 52, 867.

49 M. Atanasov, C. Daul and C. Rauzy, Struct. Bonding, 2004, 106, 97.

50 I. Ciofini and C. A. Daul, Coord. Chem. Rev., 2003, 238-239, 187.

51 R. D. Shannon, Acta Crystallogr., Sect. A: Cryst. Phys., Diffr., Theor. Gen. Crystallogr., 1976, 32, 751.

52 R. D. Cowan, The theory of atomic structure and spectra, University of California Press, Berkeley, 1997.

53 S. Hüfner, Optical spectra of transparent rare earth compounds, Academic Press, New York, 1978.

54 H. Ramanantoanina, W. Urland, F. Cimpoesu and C. Daul, Phys. Chem. Chem. Phys., 2014, 16, 12282.

55 P. S. Peijzel, P. Vergeer, A. Meijerink, M. F. Reid, L. A. Boatner and G. W. Burdick, Phys. Rev. B: Condens. Matter Mater. Phys., 2005, 71, 045116.

56 L. Esterowitz, F. J. Bartoli, R. E. Allen, D. E. Wortman, C. A. Morrison and R. P. Leavitt, Phys. Rev. B: Condens. Matter Mater. Phys., 1979, 19, 6442. 
57 M. Laroche, J.-L. Doulanm, S. Girard, J. Margerie and R. Moncorgé, J. Opt. Soc. Am. B, 2000, 17, 1291.

58 D. J. Newman and B. K. C. Ng, Crystal Field Handbook, Cambridge University Press, Cambridge, 2000.

59 C. Ronda, J. Lumin., 2002, 100, 301.

60 B. Herden, A. Meijerink, F. T. Rabouw, M. Haase and T. Jüstel, J. Lumin., 2014, 146, 302.

61 Q. Y. Zhang and X. Y. Huang, Prog. Mater. Sci., 2010, $55,353$.
62 M. Laroche, A. Braud, S. Girard, J.-L. Doualan, R. Moncorgé and M. Thuau, J. Opt. Soc. Am. B, 1999, 16, 2269.

63 D. L. Clark, J. C. Gordon, P. J. Hay and R. Poli, Organometallics, 2005, 24, 5747.

64 M. L. Neidig, D. L. Clark and R. L. Martin, Coord. Chem. Rev., 2013, 257, 394.

65 P. Dorenbos, J. Lumin., 2000, 91, 91.

66 P. Dorenbos, J. Lumin., 2000, 91, 155.

67 C. F. Schäffer, Proc. R. Soc. London, Ser. A, 1967, 297, 96. 